\title{
Immunomagnetic enrichment to evaluate the role of home environment specimens in transmission of enterovirus 71
}

\author{
WANGFENG ZHU ${ }^{1}$, XIUHUI YANG ${ }^{1,2}$, YONG ZHOU ${ }^{2}$ and YANSHENG YAN ${ }^{1,2}$ \\ ${ }^{1}$ The School of Public Health, Fujian Medical University, Fuzhou, Fujian 350004; ${ }^{2}$ Fujian Province Key Laboratory \\ of Zoonosis Research, Fujian Center for Disease Control and Prevention, Fuzhou, Fujian 350001, P.R. China
}

Received April 11, 2018; Accepted June 28, 2018

DOI: $10.3892 /$ etm.2018.6484

\begin{abstract}
Enterovirus 71 (EV71) immunomagnetic enrichment technique and routine detection methods were combined to detect swab environmental specimens to elucidate the role of environmental specimens in the spread of EV71. Immunomagnetic beads with specific enrichment of EV71 virus were prepared, then the beads were used to absorb the EV71 virus from environmental samples. Obtained immunomagnetic bead-virus complexes were detected by RT-PCR, RT-qPCR and cell culture. Isolated virus were subjected to VP1 full-length amplification and homology analysis was performed. A total of $4 \mu \mathrm{g}$ of EV71 monoclonal antibody was mixed with $50 \mu 1$ magnetic beads, and the highest coating efficiency was reached after incubating at room temperature for $2 \mathrm{~h}$. Satisfactory enrichment effect was achieved by adding $50 \mu \mathrm{l}$ immunomagnetic beads to $1.5 \mathrm{ml}$ sample and shaking at room temperature for $2 \mathrm{~h}$. The method of EV71 enrichment has high sensitivity and specificity. A total of 346 specimens after enrichment by immunomagnetic beads, the positive rates of RT-qPCR, RT-PCR and cell culture were 20.52, 5.78 , and $9.25 \%$, respectively, which were also significantly higher than those before enrichment (15.90, 3.47 and 4.05\%; $\mathrm{P}<0.05)$. After enrichment with immunomagnetic beads, isolation rate of EV71 virus from case specimens and home environment specimens increased from 27.45 to $43.14 \%$ and from 0 to $5.29 \%$, respectively. In home environment-positive specimens, positive rate of toys and stationery was high (52.00 and $24.00 \%$, respectively). In kindergarten environmental samples, the positive rate of RT-qPCR was $6.12 \%$, and EV71 virus was not isolated. Sequence analysis showed that the nucleotide homology of case isolates and home environment isolates was $98.0-100 \%$.
\end{abstract}

Correspondence to: Dr Xiuhui Yang or Dr Yansheng Yan, The School of Public Health, Fujian Medical University, 88 Jiaotong Road, Fuzhou, Fujian 350004, P.R. China

E-mail: zwfpremed@163.com

E-mail: yysh@fjcdc.com.cn

Key words: enterovirus 71, immunomagnetic beads, enrichment, environmental swab, detection

\section{Introduction}

Hand, foot and mouth disease (HFMD) is one of the most common infectious diseases in children under 5 years. Typical clinical manifestations of HFMD include mild fever, herpangina and limb herpes. A few patients with HFMD may have severe neurological symptoms, including aseptic meningitis, acute flaccid paralysis, brainstem encephalitis, and neurogenic pulmonary edema, or even death $(1,2)$. Since the 1990s, HFMD has emerged in countries in the Asia-Pacific region, and large-scale outbreaks have occurred in Taiwan, Malaysia and Singapore (3-5). In mainland China, the disease has spread throughout the country since the large-scale EV71 related HFMD outbreak in 2007 in Linyi City of Shandong Province and Fuyang City of Anhui Province in 2008 (6,7). Chinese government began to classify HFMD as a category $\mathrm{C}$ notifiable infectious disease in 2008. From 2008 to 2017, a total of 18.18 million cases of hand-foot-mouth disease were reported in mainland, including 3,632 deaths (www.chinacdc.cn).

HFMD can be caused by a variety of human enteroviruses, among which enterovirus 71 (EV71) and coxsackievirus type A (CVA) types 16, 10 and 6 are the most common. EV71 is neurotropic and causes severe nervous system infections and even death. Studies have shown that $\sim 80 \%$ of severe cases and $>93 \%$ of deaths are caused by EV71 infection (8). However, there are still no effective antiviral drugs available for anti-EV71 infection, and the use of vaccines has not been popularized. Therefore, blocking the transmission of EV71 plays an important role in controlling its infection. HFMD can be transmitted through close contact, air and other means. Contact with contaminated hands and objects can also cause transmission. Spread of HFMD through close contact has been widely confirmed (9), but transmission through environmental factors has not been well studied. Goh et al (10) found through case-control studies that exposure to contaminated household items, such as toys, stationery, tableware, toiletries, and tabletops, is a risk factor for the spread of HFMD. Li et al (11) found that contact with the hands of contaminated guardians and public playgrounds also led to the spread of HFMD. However, the above studies were only risk analysis or RT-qPCR-based assays, and isolation of EV71 from environmental samples should be performed to elucidate the homology between case isolates and environmental strains, so as to elucidate the spread of the virus. 
Because environmental specimens have the characteristics of low virus concentration and complex components, it is difficult to isolate viruses using traditional cell culture methods. The use of polymerase chain reaction (PCR) is challenged by impurities in environmental specimens, causing a false positive or false negative. PCR can be used to detect the nucleic acid of virus, but it is not able to detect virus infectivity. Immunomagnetic enrichment (IME) is an antigen-antibody-based immunological binding technique that can efficiently capture and separate intact virus particles, which in turn enables the enrichment of viruses and remove impurities. This technology combined with RT-qPCR has been successfully applied to the detection of norovirus and hepatitis A virus in complex environmental samples such as feces, food and sewage, and high sensitivity and specificity were achieved (12-14). However, there have been no reports of isolation of viruses by combination of IME and cell culture. In view of the above, in this study, immunomagnetic beads of EV71 were prepared. Combined with cell culture, RT-PCR and RT-qPCR techniques, a sensitive and effective method for EV71 virus isolation from environmental specimens was established.

\section{Materials and methods}

Reagents and instruments. EV71 virus strain (FJLY08) was donated by Dr Weng Yuwei, director of the Department of Virology, Fujian Provincial Center for Disease Control and Prevention. RD cells were provided by the Polio Laboratory of the Chinese Center for Disease Control and Prevention. Magnetic beads (11201D; Dynabeads ${ }^{\circledR}$ M-280 Sheep anti-mouse IgG; Thermo Fisher Scientific, Inc., Waltham, MA, USA) and magnetic separator were purchased from Invitrogen (Thermo Fisher Scientific, Inc.). EV71 monoclonal antibody was a gift from Professor Xia Ningshao of Xiamen University School of Public Health. AMV reverse transcriptase, random primers, Taq DNA polymerase, and RNase inhibitor were purchased from Takara Biotechnology Co., Ltd. (Dalian, China). Virus nucleic acid extraction kit and gel extraction kit were purchased from Qiagen GmbH (Hilden, Germany). EV71 nucleic acid detection kit was purchased from Jiangsu Shuo Shi Technology Co., Ltd. The study was approved by the Ethics Committee of The School of Public Health, Fujian Medical University (Fuzhou, China).

\section{Experimental methods}

The process of EV71 enrichment of using immunomagnetic beads. Magnetic beads in kit were coated with goat anti-mouse antibody (11201D, Dynabeads ${ }^{\circledR}$ M-280 Sheep anti-Mouse IgG; Thermo Fisher Scientific, Inc.). Monoclonal antibodies against EV71 were incubated with magnetic beads to form EV71 specific immunomagnetic beads (EV71-IMB). The EV71-IMB were used to capture EV71 virus to form immunomagnetic bead-virus complexes. Immunomagnetic bead-virus complex was adsorbed and washed using a magnetic separator and then resuspended to form immunomagnetic bead-virus solution for RT-qPCR, RT-PCR detection and cell culture (Fig. 1).

Preparation of EV71-IMB. According to the instructions of kit, magnetic beads were shaken well, and $50 \mu \mathrm{l}$ of the magnetic beads suspension was transferred to a $1.5 \mathrm{ml}$
EP tube, and $1 \mathrm{ml}$ washing solution (PBS, 0.1\% BSA, $2 \mathrm{mM}$ EDTA, pH 7.4) was added. The tube was placed on magnetic separator and supernatant was discarded. This procedure was repeated 3 times. Then $200 \mu$ l of antibody $(65 \mathrm{H} 12)$ was added and incubated at room temperature with shaking. Supernatant was discarded and the beads were washed 3 times with washing solution. Finally, $50 \mu \mathrm{l}$ of stock solution (PBS, $0.1 \%$ BSA, $0.05 \% \mathrm{NaN}_{3}$ ) was added and stored at $4^{\circ} \mathrm{C}$ before use.

Detection of sensitivity and specificity of EV71 enrichment using immunomagnetic beads. FJLY08 strain with a known titer $\left(10^{7.5}\right.$ TCID $\left._{50} / 100 \mu \mathrm{l}\right)$ was serially diluted in PBS and the virus diluted at $10^{2} \mathrm{TCID}_{50} / 100 \mu 1-10^{-4} \mathrm{TCID}_{50} / 100 \mu \mathrm{l}$ was enriched with EV71-IMB and detected by cell culture, RT-PCR, and RT-qPCR. The maximum dilution that can be detected by each method is the detection limit of the method. Detection was performed 3 times, and positive signals in all 3 repeats were defined as positive. Quantification by RT-qPCR

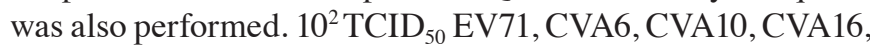
echovirus 6 , and echovirus 7 were simultaneously mixed in $1 \mathrm{ml}$ of PBS. After the mixture incubation with EV71-IMB for $2 \mathrm{~h}$, nucleic acids of immunomagnetic bead-virus complex suspension were extracted. RT-PCR amplification was performed using universal primers for enterovirus (292, 5'-MIGCIGYIGARACNGG-3'; 222, 5'-CICCIGGIGGIAYR WACAT-3'). PCR products were sequenced and analyzed.

Specimen collection and processing. When EV71 clustered cases were found, the following three types of specimens were collected: i) throat swab specimens of the cases and their guardians; ii) swab specimens of the toys, stationery, tableware, toiletries, table tops at patients home, and hands of guardian; iii) environment swab specimens such as table tops, railings, toys, and playgrounds of kindergartens with clustered cases. Throat swabs were stored in $5 \mathrm{ml}$ of MEM with 5\% FBS. Environmental swabs and hand swabs were soaked in PBS and swabbed three times in a $100-200 \mathrm{~cm}^{2}$ range and then stored in $5 \mathrm{ml}$ of PBS. All samples were stored at $4^{\circ} \mathrm{C}$ and sent to the laboratory within $12 \mathrm{~h}$. Swab specimens were mixed on a vortex shaker for $5 \mathrm{~min}$ and centrifuged at 4,000 x $\mathrm{g}$ for $10 \mathrm{~min}$. Supernatant was filtered through a $0.22 \mu \mathrm{m}$ aperture needle filter and stored at $-80^{\circ} \mathrm{C}$ before use.

Enrichment of EV71 virus in specimen using EV71-IMB. A total of $50 \mu \mathrm{l}$ of EV71-IMB was mixed with $1.5 \mathrm{ml}$ treated sample solution, followed by incubation for $2 \mathrm{~h}$ at room temperature with shaking. The tube was placed on a magnetic separator, and beads were washed 3 times with washing solution. Magnetic bead-virus complex was resuspended in $150 \mu \mathrm{l}$ MEM and stored at $4^{\circ} \mathrm{C}$ for follow-up detection. The above steps were repeated in case sample volume $>1.5 \mathrm{ml}$.

Detection of EV71 after IME. Cell culture: immunomagnetic bead-virus complex suspension was inoculated into monolayer RD cells (200 $\mu \mathrm{l} /$ well). CPE was observed daily. If no CPE was observed after 7 days, subculture was performed, followed by observation for another 7 days. If CPE still did not appear, the sample as judged as negative.

RT-qPCR and RT-PCR: RNA was extracted from $140 \mu \mathrm{l}$ of immunomagnetic beads-virus complexes using the QIAamp Viral RNA Mini kit (52906; Qiagen $\mathrm{GmbH}$ ), followed by detection of EV71 through RT-qPCR using EV71 nucleic acid detection kit (JC20102; Perfectus, Jiangsu Bioperfectus Technologies Co., Ltd., Taizhou, China). EV71-specific primer 


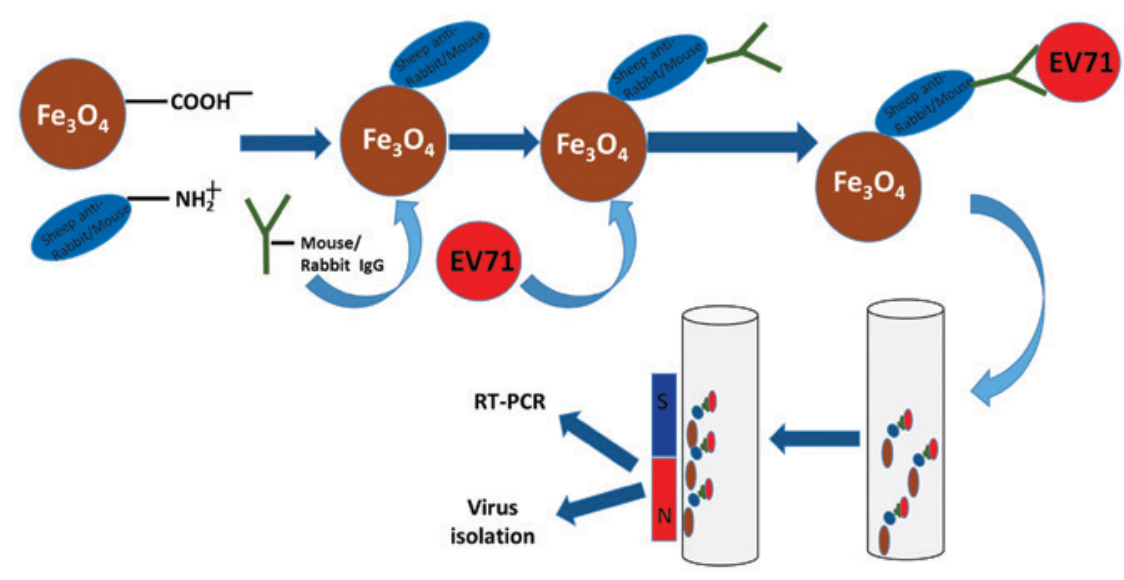

Figure 1. Procedure of enrichment of EV71 using immunomagnetic beads. EV71, enterovirus 71.

(2372, 5'-GCAGCCCAAAAGAACTTCAC-3'; 3454, 5'-AAG TCGCGAGAGCTGTCTTC-3') were used in ordinary RT-PCR to amplify full length EV71 VP1. PCR products were sequenced by Shanghai Biosune Biotechnology Co., Ltd. (Shanghai, China).

EV71 direct detection without enrichment (non-IME). Virus culture, RT-qPCR and RT-PCR were used to detect EV71 directly from sample solution without enrichment as mentioned above.

Statistical analysis. Positive rate of the two detection methods was compared using McNermar test $(\alpha=0.05)$. The comparison of rates was performed using the Pearson Chi-square test $(\alpha=0.05)$ or Fisher's exact test. Statistical analyses were performed using SPSS (version 22.0; IBM Corp., Armonk, NY, USA). Nucleotide and amino acid sequence alignment and homology analysis were performed using Bio-Edit software (V7.0.1). Phylogenetic tree was constructed by MEGA software (V6.0) using neighbor-joining method.

\section{Results}

Optimization of preparation conditions for immunomagnetic beads

Optimization of coated antibody quantity. Different amounts $(1,2,4,6,8$, and $10 \mu \mathrm{g})$ of antibody were added into an EP tube containing $50 \mu \mathrm{l}$ of magnetic beads. After incubation at room temperature for $16 \mathrm{~h}$, the amount of antibodies before and after coating was measured. Coating efficiency $=$ (amount of antibody before coating - amount of antibody after coating)/amount of antibody before coating $\times 100 \%$. As shown in Fig. 2, when the amount of antibody is $<2 \mu \mathrm{g} / 50 \mu \mathrm{l}$ of magnetic beads, the coating efficiency is $100 \%$, and as the amount of antibody increases, the coating efficiency of magnetic beads gradually decreases. Although the coating efficiency is the highest when the amount of antibody added is $<2 \mu \mathrm{g} / 50 \mu \mathrm{l}$ of magnetic beads, amount of antibodies may be insufficient. Therefore, in order to obtain the maximum coating efficiency and ensure that the amount of antibody is sufficient, $4 \mu \mathrm{g} / 50 \mu \mathrm{l}$ of magnetic beads was used in the following experiments.

Optimization of coating time. A total of $4 \mu \mathrm{g}$ of antibody was added into an EP tube containing $50 \mu \mathrm{l}$ of magnetic beads,

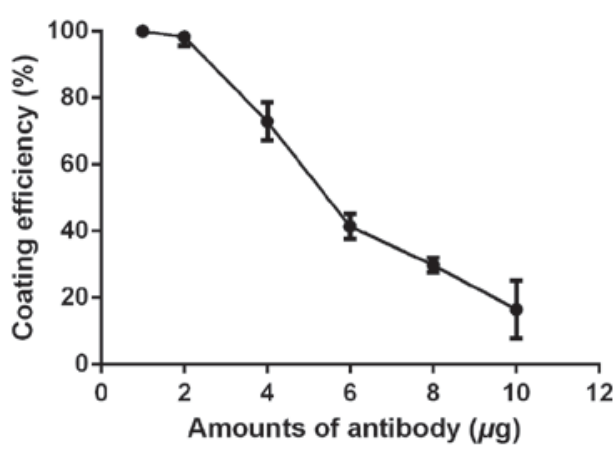

Figure 2. Optimization of coated antibody quantity.

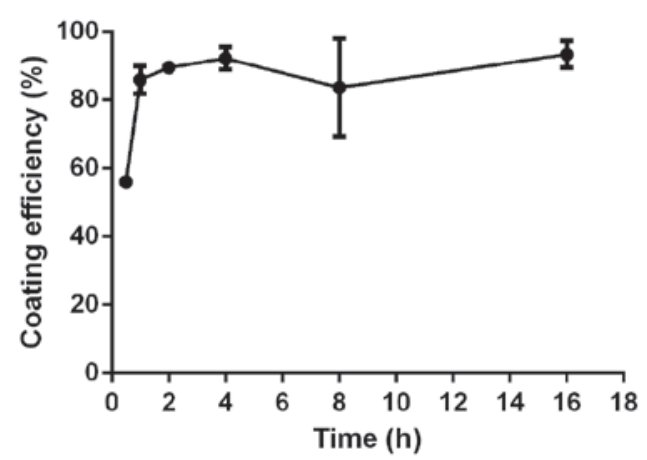

Figure 3. Optimization of coating time.

followed by incubation for $0.5,1,2,4,8$, and $16 \mathrm{~h}$, respectively. The amount of antibody before and after magnetic bead coating was measured. Coating efficiency $=$ (amount of antibody before coating - amount of antibody after coating)/amount of antibody before coating $\times 100 \%$. As shown in Fig. 3, coating efficiency increased 0.5 to $2 \mathrm{~h}$. However, after $2 \mathrm{~h}$, coating efficiency did not change significantly with the increase of the coating time. Therefore, the optimal coating time of the antibody was set at $2 \mathrm{~h}$.

Sensitivity and specificity of EV71 enrichment using immunomagnetic beads. After the enrichment of immunomagnetic beads, detection limit of cell culture was $10^{-1} \mathrm{TCID}_{50} / 100 \mu \mathrm{l}$, with an average of $5.47 \times 10^{4}$ copies $/ \mathrm{ml}(95 \%$ CI: 
Table I. Specimens collected from four epidemics of EV71 infection.

$\frac{\text { Throat swab }}{\text { Hand swab }} \frac{\text { Patients home environment swab }}{\text { Table }} \frac{\text { Kindergarten environment swab }}{\text { Table }}$

Regions Patients Guardians Guardians Toys Stationery Toiletries surface Tableware Total surface Railing Toys Playground Others Total

\begin{tabular}{lrrrrrrrrrrrrrrrrrr}
\hline Longyan & 18 & 18 & 0 & 9 & 9 & 9 & 9 & 9 & 45 & 3 & 4 & 4 & 3 & 2 & 16 \\
Xiamen & 8 & 8 & 7 & 7 & 7 & 7 & 7 & 7 & 35 & 3 & 4 & 5 & 4 & 0 & 16 \\
Ningde & 8 & 8 & 7 & 7 & 7 & 7 & 7 & 7 & 35 & 4 & 4 & 4 & 4 & 1 & 17 \\
Sanming & 17 & 17 & 11 & 11 & 11 & 11 & 11 & 11 & 55 & 0 & 0 & 0 & 0 & 0 & 0 \\
Total & 51 & 51 & 25 & 34 & 34 & 34 & 34 & 34 & 170 & 10 & 12 & 13 & 11 & 3 & 49 \\
\hline
\end{tabular}

Table II. Results of EV71 detection from different types of samples.

\begin{tabular}{|c|c|c|c|c|c|c|c|c|}
\hline \multirow[b]{2}{*}{ Sample from } & \multirow[b]{2}{*}{ Sample types } & \multirow[b]{2}{*}{ Number } & \multicolumn{3}{|c|}{ IME } & \multicolumn{3}{|c|}{ Non-IME } \\
\hline & & & RT-qPCR & RT-PCR & Cell culture & RT-qPCR & RT-PCR & Cell culture \\
\hline Patient & Throat swab & 51 & 37 & 16 & 22 & 36 & 12 & 14 \\
\hline Guardian & Throat swab & 51 & 6 & 1 & 1 & 4 & 0 & 0 \\
\hline Guardian & Hand swab & 25 & 0 & 0 & 0 & 0 & 0 & 0 \\
\hline Household item & Wipe swab & 170 & 25 & 3 & 9 & 14 & 0 & 0 \\
\hline Kindergarten & Wipe swab & 49 & 3 & 0 & 0 & 1 & 0 & 0 \\
\hline Total & & 346 & 71 & 20 & 32 & 55 & 12 & 14 \\
\hline
\end{tabular}

IME represents sample processed by IME, non-IME represents sample not processed by IME, numbers in table represent the number of tested samples and positive samples. Among throat swab samples from 51 patients, two samples were positive for IME-RT-qPCR, but negative for non-IME-RT-qPCR, one sample was positive for non-IME-RT-qPCR, but negative for IME-RT-qPCR. For the positive sample for non-IME RT-PCR and non-IME cell culture, the results of IME-RT-PCR and IME-cell-culture were also positive. IME, immunomagnetic enrichment.

3.53-7.42 $\times 10^{4}$ copies/ml). Detection limits of RT-qPCR and RT-PCR were $10^{-4} \mathrm{TCID}_{50} / 100 \mu 1$ and $1 \mathrm{TCID}_{50} / 100 \mu 1$, respectively, and the average was $3.57 \times 10^{2}$ copies $/ \mathrm{ml}(95 \% \mathrm{CI}$ : $1.36-5.79 \times 10^{2}$ copies $\left./ \mathrm{ml}\right)$ and $5.30 \times 10^{5}$ copies $/ \mathrm{ml}(95 \%$ CI: 2.81-7.78x $10^{5}$ copies $\left./ \mathrm{ml}\right)$, respectively. EV71 and 3 other common HFMD-related type (CVA6, 10, 16 group A) of virus and 2 types (echovirus 6 and 7 belong to group B) virus were mixed in equal amounts and tested by EV71 IME method. The results showed that only EV71 was detected specifically, other types of viruses were not detected. The data suggest that EV71 immunomagnetic beads can specifically enrich the EV71 virus without enriching other viruses.

\section{Practical application of imunomagnetic bead enrichment} technology

Sample collection. A total of 346 specimens were collected from four outbreaks of EV71 infection in Xiamen, Longyan, Ningde, and Sanming City. Of these, 102 were throat swab samples from HFMD patients and patients' guardians, 219 were environmental swab specimens from home and kindergarten environments and 25 were swab specimens from guardians' hand (Table I).

Test results of IME and non-IME. The above-mentioned 346 specimens were detected directly and subjected to IME followed by detection and direct detection. Results showed (Tables II and III) that, after enrichment by immunomagnetic beads, the positive rates of RT-qPCR, RT-PCR, and cell culture were $20.52,5.78$ and $9.25 \%$, respectively, which were significantly higher than those of direct detection $(15.89,3.47$ and $4.05 \%$, respectively) $(\mathrm{P}<0.05)$. In the case specimens, the positive rate of cell culture after enrichment using magnetic beads was $43.14 \%$, which was significantly higher than that of direct cell culture $(27.45 \%)(\mathrm{P}<0.05)$. In household product samples, EV71 was not isolated directly from the cell culture, but the isolation rate of EV71 after enrichment with immunomagnetic beads was $5.29 \%(9 / 170)$. The positive rates of RT-qPCR was significantly increased from 8.24 to $14.71 \%(\mathrm{P}<0.05)$.

Detection of EV71 in different environment specimens. In environmental samples, positive results of one of the three detection methods after enrichment were considered as positive. Positive rates of environmental swabs from home and kindergarten were $14.71 \%(25 / 170)$ and $6.12 \%$ (3/49), respectively, and there was no significant difference between them $(\mathrm{P}>0.05)$. In home environment specimens, positive rates for toys, stationery, toiletries, tableware, and tabletops were $38.23 \%$ (13/34), 17.65\% (6/34), 5.88\% (2/34), and $5.88 \%(2 / 34)$ and $5.88 \%(2 / 34)$, respectively, and there were significant differences among them $(\mathrm{P}<0.05)$. Most of the 25 positive specimens were detected in toys and stationery, of which 13 were toys $(52.00 \%)$ and 6 were stationery (24.00\%). In kindergarten environmental samples, there was no difference in the positive rate among different environmental samples $(\mathrm{P}>0.05)$ (Table IV). 
Table III. Comparison of EV71 detection results between IME and non-IME.

\begin{tabular}{|c|c|c|c|c|c|}
\hline \multirow[b]{2}{*}{ Sample } & \multirow[b]{2}{*}{ Method } & & \multicolumn{2}{|c|}{ Non-IME } & \multirow[b]{2}{*}{ McNemar test } \\
\hline & & & Positive & Negative & \\
\hline \multirow[t]{6}{*}{ Patient } & RT-qPCR & Positive & 35 & 2 & \\
\hline & & Negative & 1 & 13 & \\
\hline & RT-PCR & Positive & 11 & 5 & $\chi^{2}=1.500, P>0.05$ \\
\hline & & Negative & 1 & 34 & \\
\hline & Cell culture & Positive & 14 & 8 & $\chi^{2}=6.125, P<0.05$ \\
\hline & & Negative & 0 & 29 & \\
\hline \multirow[t]{6}{*}{ Household item } & RT-qPCR & Positive & 11 & 14 & $\chi^{2}=5.882, P<0.05$ \\
\hline & & Negative & 3 & 142 & \\
\hline & RT-PCR & Positive & 0 & 3 & \\
\hline & & Negative & 0 & 167 & \\
\hline & Cell culture & Positive & 0 & 9 & \\
\hline & & Negative & 0 & 161 & \\
\hline \multirow[t]{6}{*}{ Total } & RT-qPCR & Positive & 54 & 17 & $\chi^{2}=12.5, \mathrm{P}<0.0001$ \\
\hline & & Negative & 1 & 274 & \\
\hline & RT-PCR & Positive & 11 & 9 & $\chi^{2}=4.9, P=0.021$ \\
\hline & & Negative & 1 & 325 & \\
\hline & Cell culture & Positive & 14 & 18 & $\chi^{2}=16.05, \mathrm{P}<0.0001$ \\
\hline & & Negative & 0 & 314 & \\
\hline
\end{tabular}

IME, immunomagnetic enrichment.

Table IV. The detection results of EV71 in environmental samples via IME.

\begin{tabular}{|c|c|c|c|c|c|}
\hline \multirow[b]{2}{*}{ Sample from } & \multirow[b]{2}{*}{$\begin{array}{c}\text { Sample } \\
\text { types }\end{array}$} & \multirow[b]{2}{*}{ Number } & \multicolumn{3}{|c|}{ Detection } \\
\hline & & & Positive & Negative & $\begin{array}{c}\text { Fisher's } \\
\text { exact test }\end{array}$ \\
\hline \multirow{5}{*}{$\begin{array}{l}\text { Household } \\
\text { item }\end{array}$} & Toy & 34 & 13 & 21 & $\mathrm{P}<0.05$ \\
\hline & Stationery & 34 & 6 & 28 & \\
\hline & Toiletry & 34 & 2 & 32 & \\
\hline & Tableware & 34 & 2 & 32 & \\
\hline & Tabletop & 34 & 2 & 32 & \\
\hline \multirow[t]{6}{*}{ Kindergarten } & Toy & 13 & 1 & 12 & $P>0.05$ \\
\hline & Tabletop & 10 & 0 & 10 & \\
\hline & Handrail & 12 & 0 & 12 & \\
\hline & Playground & 11 & 1 & 10 & \\
\hline & Others & 3 & 1 & 2 & \\
\hline & Total & 219 & 28 & 191 & \\
\hline
\end{tabular}

All samples were processed by IME. IME, immunomagnetic enrichment.

Homology of EV71 isolated from environment and patients. Full-length VP1 was amplified from EV71 obtained from patients, guardians, and environment samples and was sequenced. Results showed (Table V) that the nucleotide and amino acid homology of the virus strains in 4 outbreaks of 4 regions were $92.8-100 \%$ and $97.9-100 \%$,
Table V. Comparison of nucleotide and amino acid homology of EV71 isolates in different regions.

\begin{tabular}{lcccr}
\hline & \multicolumn{4}{c}{ Nucleotide homology (\%) } \\
\cline { 2 - 5 } & Xiamen & Ningde & Longyan & Sanming \\
\hline Xiamen & 100 & & & \\
Ningde & 92.8 & 100 & & \\
Longyan & $93.7-93.8$ & $96.1-96.2$ & $99.8-100$ & \\
Sanming & $94.0-94.6$ & $96.6-96.9$ & $97.4-98.3$ & $98.0-100$ \\
\hline & \multicolumn{4}{c}{ Amino acid homology (\%) } \\
\cline { 2 - 5 } & Xiamen & Ningde & Longyan & Sanming \\
\hline Xiamen & 100 & & & \\
Ningde & 98.9 & 100 & & \\
Longyan & $97.9-98.3$ & $98.9-99.3$ & $99.6-100$ & \\
Sanming & 98.6 & 99.6 & $99.3-99.6$ & \\
\hline
\end{tabular}

respectively. Environmental strains and case strains obtained in the same epidemic in the same area were closely related. Nucleotide and amino acid homology was $98.0-100 \%$ and 99.6-100\%, respectively, indicating that the environmental strains and the case strains were in the same transmission chain. Phylogenetic tree also showed similar results. The strains in the four regions belonged to the same branch and all belonged to the $\mathrm{C} 4 \mathrm{a}$ subtype. Strains isolated from the 


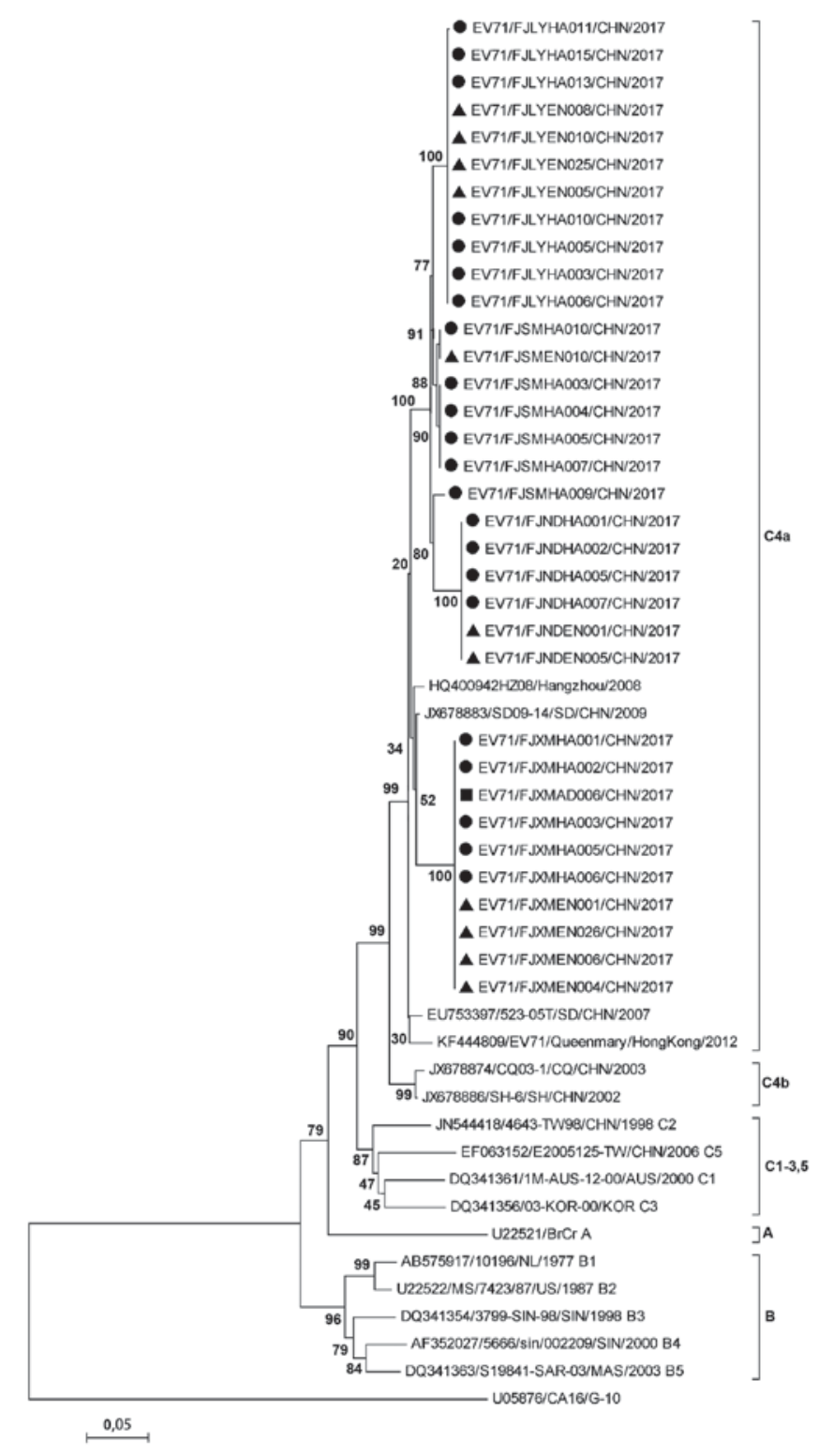

Figure 4. Phylogenetic tree constructed based on partial VP1 nucleotide sequences of EV71. Phylogenetic tree was constructed using the neighbor-joining method based on the alignment of the partial VP1 region sequences of Fujian strains and other strains downloaded from GenBank. Bootstrap values for 1,000 replicates were calculated. ${ }^{*}$ Clinical strains, ^household strains, "scaregiver strain. CA16 strain (U05876) was used as an out-group. EV71, enterovirus 71.

same area were all grouped in the same cluster, except that EV71/FJSMHA009/2017 belonged to the Ningde isolate cluster. But the homology of the EV71/FJSMHA009/2017 strain with other Sanming strains was $98.0 \%$, and with the Ningde strains was $96.9 \%$. Among clustered cases in Xiamen, the EV71/FJXMEN001/CHN/2017 strain was isolated from children's toy specimens, and EV71/FJXMHA001/CHN/2017 and EV71/FJXMHA002/CHN/2017 strains were isolated from sibling throat swabs, respectively. These three viruses were derived from the same family, and their nucleotide homology was $100 \%$, indicating the presence of family cross-infection. EV71/FJXMAD008/CHN/2017 was the isolate of throat swab specimens from other cases of guardian, and its homology with the isolates in Xiamen was $100 \%$ (Fig. 4).

\section{Discussion}

EV71 virus can be excreted with the excreta of infected persons, respiratory tracts and skin rashes. Results of epidemiological studies have clearly shown that articles contaminated with excreta or secretions from children can cause indirect contact infection, and experimental studies have found that these contaminants indeed contain virus-associated antigens or nucleic acids, but no live virus has been isolated from these contaminants $(10,11)$. It is difficult to isolate live virus from contaminated environmental samples, mainly due to the low concentration of virus in environmental specimens, which is lower than the detection limit of conventional detection methods. Complex components in environmental specimens contain impurities that interfere with subsequent cell culture or RT-PCR assays. For example, organic substances can interfere with PCR and prevent the template from being amplified, thus causing false negative or false positive results. Therefore, finding effective ways to concentrate, enrich viruses, and remove impurities is the key to virus detection in environmental samples.

IME technology is based on the antigen-antibody immune binding reaction. This technique only isolates antigenically intact virus particles, and rarely adsorb naked nucleic acid (13). Therefore, IME technology can simultaneously detect the antigenicity and nucleic acid of the virus for the assessment of the risk of virus infection. Compared with common PCR methods, IME technology has a higher degree of confidence, and it also provides a more effective tool for assessing the infection risk of viruses that cannot be cultivated yet. The EV71 monoclonal antibody immunomagnetic beads prepared in this study have high specificity. After enriching the immune magnetic beads, the antigen-antibody complexes need not be separated from the magnetic beads, and the cells can be directly inoculated without affecting the normal cell growth. Cytopathic effect caused by virus infection was observed, indicating that this enrichment method does not affect the virus infectivity.

EV71 can remain in the intestine and upper respiratory tract for 2-4 weeks. Therefore, specimens such as feces, rectal swabs, and throat swabs are often used for virus detection and isolation. Among them, the throat swab specimens are considered to be the most appropriate specimens for HFMD detection because of their high detection rate and convenient sampling, and are suitable for outpatient and inpatient cases (15). In this study, the positive rate of virus isolation was significantly higher in cases of throat swabs enriched with immunomagnetic beads compared to direct cell culture. This shows that the combination of immunomagnetic beads enrichment and cell culture can increase the detection rate of case pathogens. Therefore, in the outbreak of HFMD, immune magnetic bead enrichment will be an effective tool for virus isolation. National Center for Immunization and Respiratory Diseases of the United States found that adults with latent infection do not show any clinical symptoms, but they can still spread the virus to others (http://www.cdc. gov/hand-fo-mouth/About/Transmission.html), and they are very important sources of infection. In this study, the EV71 virus was detected in child guardians from throat swab. The guardian isolate has $100 \%$ homology with the case isolate. 
This suggests that child guardians can serve as vehicles for the transmission of EV71 and play an important role in the transmission of EV71. Considering that adult infections are mostly asymptomatic and the range of activities of adults is much greater than that of children, it is very important to strengthen monitoring of guardians.

In outbreak of EV71 infection, kindergartens are the main control site. Closing kindergartens and performing comprehensive disinfection are considered to be the most effective control measures. This study failed to separate EV71 from kindergarten specimens, which may be related to the large-scale disinfection of kindergartens before the collection of environmental specimens. Disinfection is well planned and strictly executed in Chinese kindergartens. EV71 virus was detected in part of kindergarten environmental swabs through IME-RT-qPCR, suggesting that toys and other articles are indeed contaminated with the virus. If they are not disinfected in time, the spread of the disease may occur.

In outbreaks of HFMD, the family sanitation and disinfection work is more easily overlooked. In this study, viral nucleic acids were detected in environmental specimens by RT-qPCR after IME. Positive rate was significantly increased, and the virus was isolated, revealing that immunomagnetic beads enrichment technology can improve detection sensitivity. Nucleotide sequences of the isolates from family environment specimens and case isolates were highly consistent, and the same strains were isolated in the siblings and toys of the same family, indicating that the EV71 can be spread through family cross-infection. EV71 has a high detection rate in home environment specimens, especially in toys and stationery. Therefore, in the prevention of HFMD epidemic, disinfection of the family environment should also be performed.

In conclusion, we established EV71 immunomagnetic bead enrichment technique, and combination of this technique and routine detection methods improved the detection sensitivity of EV71. This method is applied to the detection of peripheral environmental swabs in children and viruses can be isolated from environmental samples. Viruses isolated from environmental specimens and case specimens were highly homologous. It was confirmed that children's toys and stationery played an important role in the transmission of EV71.

\section{Acknowledgements}

We would like to extend our deep gratitude to the laboratory support from Department of Immunization Planning, Fujian Center for Disease Control and Prevention. We are grateful for the support during the sampling work from Qianjin Chen, Yuanhui Xiao, Heshen Chen and Daobin Zhu, the directors of Fujian Municipal Center for Disease Control and Prevention.

\section{Funding}

This study was supported by Fujian Health System Youth Backbone Talents training project 'environmental sewage applied to enterovirus epidemic rule' (2013-ZQN-ZD-10) and leading (key) project of social development in Fujian Province: changes in the epidemic of enterovirus and prevention and control strategy of PVII vaccine strain in the population and the external environment after the polio vaccine conversion (2017Y0011).

\section{Availability of data and materials}

The datasets used and/or analyzed during the present study are available from the corresponding author on reasonable request.

\section{Authors' contributions}

WZ drafted the manuscript. WZ, XY and YZ were mainly devoted to specimen collection and processing. WZ and XY helped with enrichment of EV71 virus in specimen using immunomagnetic beads. WZ, XY and YY were responsible for the conception and design of the study. All authors have read and approved the final manuscript.

\section{Ethics approval and consent to participate}

The study was approved by the Ethics Committee of The School of Public Health, Fujian Medical University (Fuzhou, China).

\section{Patient consent for publication}

Not applicable.

\section{Competing interests}

The authors declare that they have no competing interests.

\section{References}

1. Ooi MH, Wong SC, Lewthwaite P, Cardosa MJ and Solomon T: Clinical features, diagnosis, and management of enterovirus 71. Lancet Neurol 9: 1097-1105, 2010.

2. Yi EJ, Shin YJ, Kim JH, Kim TG and Chang SY: Enterovirus 71 infection and vaccines. Clin Exp Vaccine Res 6: 4-14, 2017.

3. Ho M, Chen ER, Hsu KH, Twu SJ, Chen KT, Tsai SF, Wang JR and Shih SR; Taiwan Enterovirus Epidemic Working Group: An epidemic of enterovirus 71 infection in Taiwan. N Engl J Med 341: 929-935, 1999.

4. Herrero LJ, Lee CS, Hurrelbrink RJ, Chua BH, Chua KB and McMinn PC: Molecular epidemiology of enterovirus 71 in peninsular Malaysia, 1997-2000. Arch Virol 148: 1369-1385, 2003.

5. Chan KP, Goh KT, Chong CY, Teo ES, Lau G and Ling AE: Epidemic hand, foot and mouth disease caused by human enterovirus 71, Singapore. Emerg Infect Dis 9: 78-85, 2003.

6. Zhang Y, Tan XJ, Wang HY, Yan DM, Zhu SL, Wang DY, Ji F, Wang XJ, Gao YJ, Chen L, et al: An outbreak of hand, foot, and mouth disease associated with subgenotype C4 of human enterovirus 71 in Shandong, China. J Clin Virol 44: 262-267, 2009.

7. Yang F, Ren L, Xiong Z, Li J, Xiao Y, Zhao R, He Y, Bu G, Zhou S, Wang J, et al: Enterovirus 71 outbreak in the People's Republic of China in 2008. J Clin Microbiol 47: 2351-2352, 2009.

8. Xing W, Liao Q, Viboud C, Zhang J, Sun J, Wu JT, Chang Z, Liu F, Fang VJ, Zheng Y, et al: Hand, foot, and mouth disease in China, 2008-12: An epidemiological study. Lancet Infect Dis 14: 308-318, 2014.

9. Park SK, Park B, Ki M, Kim H, Lee K, Jung C, Sohn YM, Choi SM, Kim DK, Lee DS, et al: Transmission of seasonal outbreak of childhood enteroviral aseptic meningitis and hand-foot-mouth disease. J Korean Med Sci 25: 677-683, 2010. 
10. Goh KT, Doraisingham S, Tan JL, Lim GN and Chew SE: An outbreak of hand, foot, and mouth disease in Singapore. Bull World Health Organ 60: 965-969, 1982.

11. Li P, Li T, Gu Q, Chen X, Li J, Chen X, Chen Y, Zhang D, Gao R, He Z, et al: Children's caregivers and public playgrounds: Potential reservoirs of infection of hand-foot-and-mouth disease. Sci Rep 6: 36375, 2016.

12. Casas N and Suñén E: Detection of enteroviruses, hepatitis A virus and rotaviruses in sewage by means of an immunomagnetic capture reverse transcription-PCR assay. Microbiol Res 157: $169-175,2002$

13. Liu P, Kim M, Schlesinger D, Kranz C, Ha S, Ha J, Slauch J, Baek S and Moe C: Immunomagnetic separation combined with RT-qPCR for determining the efficacy of disinfectants against human noroviruses. J Infect Public Health 8: 145-154, 2015.
14. Kobayashi S, Natori K, Takeda N and Sakae K: Immunomagnetic capture RT-PCR for detection of norovirus from foods implicated in a foodborne outbreak. Microbiol Immunol 48: 201-204, 2004

15. Ooi MH, Solomon T, Podin Y, Mohan A, Akin W, Yusuf MA, del Sel S, Kontol KM, Lai BF, Clear D, et al: Evaluation of different clinical sample types in diagnosis of human enterovirus 71-associated hand-foot-and-mouth disease. J Clin Microbiol 45: $1858-1866,2007$

(7) (9) This work is licensed under a Creative Commons

c) Attribution-NonCommercial-NoDerivatives 4.0 International (CC BY-NC-ND 4.0) License. 